\title{
App Interface Icon Design Based on Information Theory
}

\author{
Ya Sun \\ Xiamen Academy of Arts and Design \\ Fuzhou University \\ Xiamen, China 361000
}

\begin{abstract}
The science and technology progress leads to more and more clear division of labor in the design industry. UI design, as a hot major in recent years, has been gradually improving its theoretical basis. From the perspective of information theory, this paper attempts to make preliminary analysis the elements of APP icon design and their correlations.
\end{abstract}

Keywords-information theory; information transmission; APP icon; symbol

\section{INTRODUCTION}

Since the 21 st century, with the rapid development of the internet in China, high-tech electronic products such as cellphones emerge at the right moment and bring new design platforms, media, methods and styles to the field of visual communication. With the popularity of intelligent terminal products, cellphones have become the most popular terminal products, whose importance in UI design has become increasingly prominent. The UI (abbreviation of User Interface) is the abbreviation of user interface. Cellphone UI design mainly includes user research, interactive design and interface design, among which the interface design has become an important category in the field of visual communication. From the perspective of information theory, this paper analyzes the existing APP icons and discusses how to apply information theory and its methodology to the design of APP icons, so as to design APP icons with higher ratio of transmission of effective information.

\section{CONCEPT OF APP ICON DESIGN}

APP (abbreviation of Application) refers to the third party application for intelligent terminal devices, commonly known as the cellphone software and used to make up for the deficiency of the original system and improve individuality so as to offer more comprehensive and people-oriented services to people. In a sense, the APP icon is the symbol of the application and the graphical representation of the application functional information.

\section{APP ICON DESIGN BASED ON INFORMATION THEORY}

\section{A. Elements and Process of Information Transmission in APP Icon Design}

According to the information theory, the transmission process of information shall include the information source, information sink, message, information channel and feedback from the information receiver. In the design of APP icons, the designer is the information source, that is, the main body of information transmission. The cellphone user is the information sink, that is, the information receiver. The designer is required to encode the information (massage) of the APP function and transmit it to the cellphone user by using the APP icon as the information channel, so that the user can decode the encoded content, thus obtaining the information that the designer wants to convey.

\section{B. Information in APP Icon Design}

1) Semantic information: The basic content of an APP icon is to encode and integrate the semantic information (i.e. the functional information of the APP) into an icon which shall be easy to be decoded by the user. The designer encodes the information through graphics, texts, colors and other elements to form an APP icon, and the user decodes the icon according to their cognitive style, habit and experience, so as to understand the functions of the APP. For example, the icon, as shown in "Fig. 1", represents a APP with camera function, and in order to distinguish it from other camera software, it also contains another semantic information, that is, M. M refers to the Musemage, the English name of the APP.

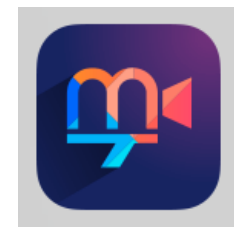

Fig. 1. MuseMage APP

2) Type information: Type information refers to the design image, colors, text, texture and image composition, 
etc., which is the technical information level in the works design. So far, there are two styles of APP icons, namely, skeuomorphic icons and flat icons. Skeuomorphic design means to be as similar as possible to the real object in the most intuitive impression, so that people can recognize the information content within a short time. Flat design means to use abstract, simplified and symbolic design elements to express the information without the highlight, shadow and perspective. For example, both "Fig. 2" and "Fig. 3" show the e-mail icons. Compared with the flat icon in Figure 3, the icon in "Fig. 2" has richer colors and the stereoscopic impression and perspective in it are easier to be accepted by people visually. The combination of opened drawer and the envelope express the concept "mailbox" in a more overall way. "Fig. 3" shows a simple flat icon which uses simple envelope image to express "mail". According to the above analysis, we can find that a skeuomorphic icon usually expresses richer information than a flat icon.

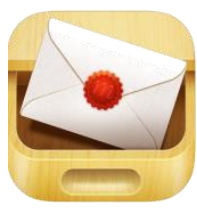

Fig. 2. Skeuomorphic APP

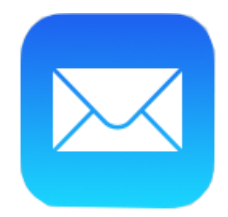

Fig. 3. Flat APP

3) Aesthetic information: Aesthetic information is the aesthetic individuality the designer conveys when conveying the functional information of the APP, which is also the aesthetic standard, emotional taste, design features and even the individual style of the designer. In the meantime, the icon of the interactive interface designed by the designer is not as a pure artistic work but designed to serve for users, so the style of an APP may also be designed specific for the preference of a certain kind of users. A successful APP icon can not only accurately convey the functional information of the APP, but also make the APP stand out among similar APPs. "Fig. 4" shows a group of icons with Chinese characteristics. There is a kite image in the game APP icon; the mail APP icon contains traditional Chinese characters and a peach twig that stands for one missing its distant relatives and friends in ancient times; in the time APP icon, the hands of the clock are replaced by bamboo leaves. All these elements express the Chinese classical mood. "Fig. 5" shows a group of highly recapitulative APP icons designed by extracting the functions and visual elements of these APPs. Theses icons are intended to mainly convey functional information of the
APP and barely the personal style characteristics of the designer.

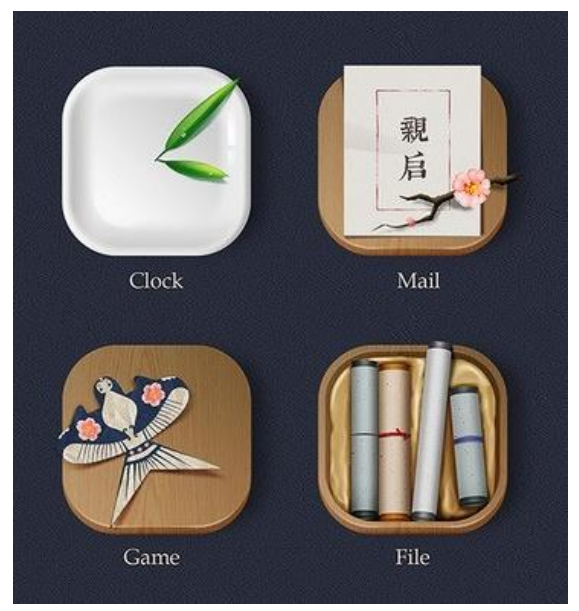

Fig. 4. APPs with Chinese characteristics.

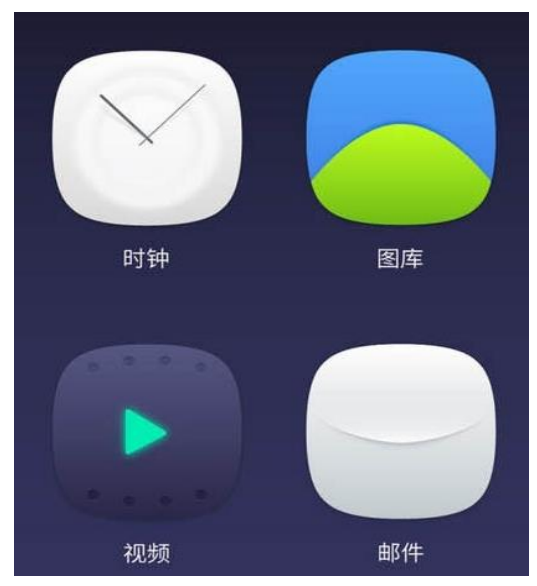

Fig. 5. APPs of simple style.

\section{CODING THEORY IN APP ICON DESIGN}

Whether the information conveyed by the APP icon designed can be accepted and recognized by users quickly, correctly and efficiently is the key point to evaluate whether the APP icon design is effective. This key point is determined depending on the designer's accuracy of mastering and using the content, image and representation form in the coding process for icon design. An excellent APP icon design should be able to covert the functional information of the APP into symbols suitable for communication, easy to be recognized, accepted and understood by users, that is, image and texts.

\section{A. Information, Signals, Symbols and APP Icon}

In any form of communication activity, information is attached to the carrier by means of various symbols to enter the communication process, so we can see that symbols are the basis of information transmission. Information is one of the necessary conditions for human to carry out 
communication activities, including artificial information and natural information. Natural information is attached to various objective things whose existence people can directly feel through such natural information and based on this, people can associate the external features of the things they have contacted. Artificial information is the product people created through artificial processing and transformation, which cannot attach to natural objects and takes symbols as their carrier.

Symbols come with the human origins and will be preserved in a relatively stable form if used at a certain frequency and accepted by all. APP icon design can only realize the communication between the designer and users and realize the equivalence of exchanged information by using these widely accepted symbols. Symbols can be created and are endowed with meanings by human beings. Some symbols are time sensitive and will change with time. The symbols such as image and text used in the APP icon are the symbols that the designer integrates and converts the information into to conform to the cognitive habits and psychological needs of users and widely accepted by users.

\section{B. Types of APP Icons and Characteristics of Information Transmission}

1) Image icon: Image symbols, through visual imitation or graphical implementation, use the things that already have meaning to express meaning. Having some similarities with daily things is the characteristic of image symbols. Therefore, image icons are generally imitations of objective things. Such kind of icons is often the most straightforward in conveying the functional information of the APPs. For example, the clock, memo, etc. icons can convey the functional information of the APPs to users in an easy way.

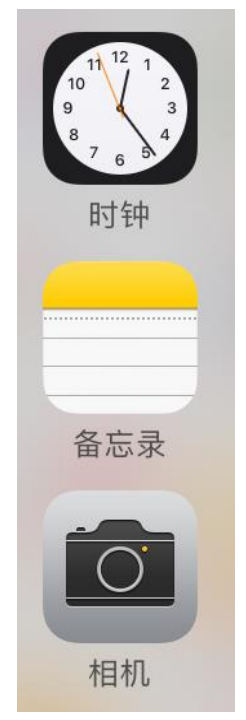

Fig. 6. ios 11 icons (1).

2) Referential icon: The semiologists put forward that a referential symbol refers to the association between the presentative object and the referent through association and inference, and such association is indirect. In general, referential icons have the characteristics of referential symbols, which are usually used to participate in information conversion, so that users can obtain the meaning and purpose of the APP through association and inference. For some referential icons, their association is relatively simple, such as the icons in "Fig. 7", indicating weather, data and stock market, respectively. For such icons, they use partial image to indicate the whole to guide people to think. Cloud will remind people of weather, tree diagram will remind people of data and zigzag line will remind people of stock. Some other icons require users to make deep thinking such as the massage icon. Compared with other image icons such as clock and memo icons, referential icons generally refer to relatively abstract things, which are usually behaviors or objects difficult to be imitated and suitable for generalization. The text message itself is not a concrete object that can be intimated. The massage icon ("Fig. 8") adopts the dialog box as the main image, which needs people to associate and decode the icon so as to understand the meaning of the icon. Compared with the image icons, referential icons need much more of users' active participation in association and inference, so the referential icons involve more complicated visual coding process, which requires the designer to think deeply about how to facilitate users' decoding.

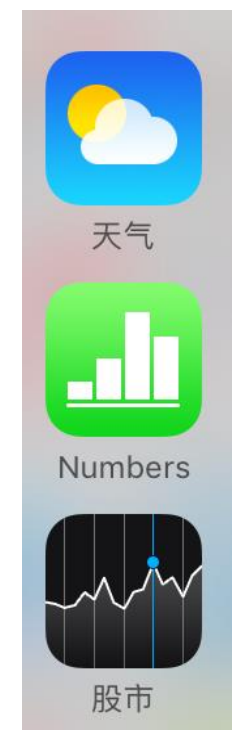

Fig. 7. ios icons (2).

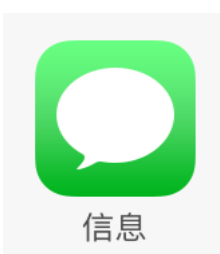


Fig. 8. ios 11 icon (3).

3) Symbolic icons: A symbol mark means that there is no necessary connection between the presentative object and the referent, but they associate with each other by depending on the social contract. A symbol mark generally has no relation with its representative object, and the symbolic relation between them is formed when the symbol mark is endowed with a certain meaning by people. Such meaning is closely associated with tradition and culture. Numbers, languages, cultural patterns, signs can be classified as symbol marks. Therefore, symbolic icons also have such characteristics, which need to take specific cultural context as basis or a general symbolic relationship that has been accepted by people for a long time. "Fig. 9" shows the logo of Tencent QQ, a social networking APP, but its icon has nothing to do with making friends or chatting. The penguins icon has been used and accepted by people for a long time, thus is recognized as the representative of QQ.

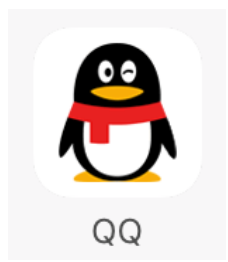

Fig. 9. Tencent qq APP.

\section{APP ICON DESIGN OPTIMIZATION PRINCIPLE BASED ON INFORMATION THEORY}

\section{A. Principle of Correct Information Coding}

APP icon design is not the form and carrier of pure artistic creation of the designer. Its main function is to convey the functional information of the APP by encoding the information depending on the representation of the icon and then deliver the information to the user correctly so as to realize the purpose of information transmission. Therefore, in APP icon design, the designer should take accurate information transmission as the primary goal. APP icon design is an attractive way of representation to optimize, reencode and organize the information. Both the design form and coding method serve the goal of conveying specific information to receivers, so that the information content can be better accepted by the receivers in the process of information dissemination. Therefore, the icon cannot be designed only for the design. However, nowadays, to express the so-called "personality" and "creativity", many designers ignore the principle of correct information transmission. For example, in "Fig. 10", there is an icon of a photo processing APP, but when users first see the icon, the first image they see is a whale which has nothing to do with the photo or photo processing. APP icons like this that do not match their functions will make users misunderstand the APPs, so they are unsuccessful design although aesthetic.

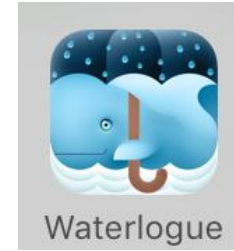

Fig. 10. Waterlogue Camera APP.

\section{B. Principle of Information Effect Enhancement}

APP icon design can enhance the recognition, propagation and influence degree of information in the icon. In addition, in the progress of APP icon design, the manifestation mode of information may convert into the material images from abstract images, and this phenomenon can avoid information content reduction due to the cognitive difference between the designer and users, so this design method can make the information easier to be accepted by the users and realize the purpose of increasing information content. For instance, the Baidu Map APP icon (shown in "Fig. 11") directly adopts the combination of map and landmark elements. Compared with Chinese characters, such simple graphs easy to recognize can help foreign users quickly and accurately accept and understand the information so as to realize the purpose of barrier-free transmission of information.

\section{Principle of Facilitating Users' Decoding}

In information accepting activities, there is communication between cellphone users and APP icons, and it is the greatest desire of the designer that the users can correctly decode the icon information (the functional information of the APP) and can subconsciously understand the designer's information intention so as to have "resonance" with the designer. Only when the icons meet the psychological expectations of the information receivers and have aesthetic perception, can users' decoding form. "Fig. 12" shows the icons of Pics Art APP in different periods. Compared with the icon in "Fig. 11", that in "Fig. 12.2" is richer in color, and the gradually varied colors make the APP more high-end, and there is no other redundant information except the deformation of simple "P" symbol. Such concise information and aesthetically pleasing icons can meet the psychological needs and aesthetics standards of users, thus facilitating users' decoding behavior.

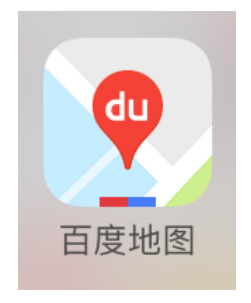

Fig. 11. Baidu Map APP. 


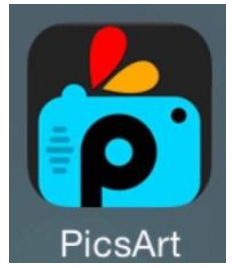

Fig. 12. 1 irst version of PicsArt (1).

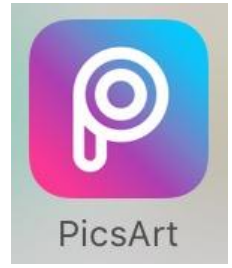

Fig. 12.2 The current version of PicsArt.

\section{Principle of Intrinsic Unified Relevance}

In the process of information transmission, all elements are interrelated and interact with each other. In the APP icon design, only when the designer (information source) and users (information sink) have similar cognitive habits and knowledge structure can an information transmission environment be created. If there is no connection between them, the effect of information dissemination will be hampered. Sago Mini in "Fig. 13" is a puzzle game for preschoolers, whose icon adopts the image of a puppy which is a favorite of this age group. This kind of coding based on the users' psychology links all elements including the information source, information sink, message, coding and decoding together, contributing to the generation of the whole information process.

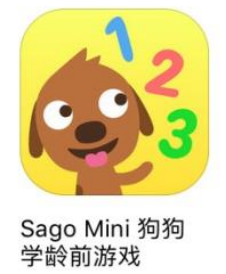

Fig. 13. Sago Mini Game APP.

\section{APP ICON DESIGN METHOD UNDER THE PRINCIPLE OF INFORMATION OPTIMIZATION}

The information we receive through our sense organ is complicated, so if we want to convey useful information to our users, we need to extract it first. Useful information is like the acceptable specific band in radio communication, while other useless information is like noise. In the icon design, first we need to analyze and classify the information, grasp the core and useful information, screen out and filter the ambiguous information to construct a reasonable and effective icon.

\section{A. Extracting Information}

People's uncertainty about one thing is because their cognition about it is not clear enough, and their fuzzy memory of information is because the information lacks emphasis. Therefore, to make the icon clear enough and focus on the point, the APP icon design shall not take care but shall focus on one point so as to realize direct transmission and reduce disturbance. Before design, the designer shall make in-depth analysis and research to understand the needs of audience in advance and clarify the information receivers, which is the basic preparation for APP icon design in the early stage. In design, the design shall, through analysis and extraction, grasp the most representative attributes and characteristics of information as the core of expression and the highlight point. The content after being repeatedly stressed will be the design theme in this information chain. Then, the construction of the symbol system shall center on and serve this theme. Only in this way can the information be received and clearly remembered by the audience.

\section{B. Redesigning Information}

The ultimate goal of APP icon design is to effectively convey the functional information of the APP. To achieve this goal, the designer shall choose an appropriate presentation method. Graphical information has both text and data and logic, thus becoming an important form of communication. Such information transformation can effectively avoid written and language barriers to make the information become more vivid and intuitive as well as effectively improving the communication efficiency.

\section{CONCLUSION}

The technological development drives the upgrading of digital products, and also generates more and more information transmission and expression methods, which gives more space for the development of mobile terminal interface. The instinctive communication of human is realized depending on collaboration of multiple sense organs, so a single communication channel will inevitably reduce the communication efficiency. Multi-channel digital terminal interface is an ideal way to convey information and also an important symbol of artificial intelligence. No matter what kind of technology is used, the more human-based interactive mode with multi-channel composite communication combination will be the developing trend in the future. Nowadays, APP icons are only presented with static images. Compared with static icons, dynamic icons can convey more semantic and aesthetic information and can attract more users' attention in the same time. How to integrate the hearing, touch, smell and other senses (such as music, dynamic and handfeel) apart from visual sense to attract users still needs the crossover cooperation between designers and technical engineers.

\section{REFERENCES}

[1] Song Liqing, Li Shiguo. APP Innovation Design with "Zodiac" Theme [J]. Packaging Engineering, 2014, 35(2): 54-58. 
[2] Han Congyao. Image: Rediscovery of Post-Semiotics [M]. Nanjing: Nanjing University Press, 2010.

[3] Hu Fei. Basis of Art Design Symbols [M]. Beijing: Tsinghua University Press, 2013.

[4] Teng Zhaoxuan, Jin Songwen, Zhen Yongliang. On the Visibility of Graphical User Interface Icon Design of Mobile Phone [J]. Packaging Engineering, 2013, 34(2): 66-70. 